\title{
Minimum time constrained control of acid strength on a sulfuric acid alkylation unit
}

\author{
N. Petit ${ }^{\mathrm{a}}$, Y. Creff ${ }^{\mathrm{b}, *}$, L. Lemaire ${ }^{\mathrm{c}}$, P. Rouchon ${ }^{\mathrm{d}}$ \\ ${ }^{a}$ Centre Automatique et Systèmes, École Nationale Supérieure des Mines de Paris, 60, boulevard Saint-Michel, 75272 Paris Cedex 06, France \\ ${ }^{\mathrm{b}}$ Elf Antar France, Centre de Recherches Elf à Solaize, BP 22, 69360 Solaize Cedex, France \\ ${ }^{c}$ Elf Antar France, Raffinerie de Feyzin, BP 6, 69551 Feyzin Cedex, France \\ ${ }^{\mathrm{d}}$ Centre Automatique et Systèmes, École Nationale Supérieure des Mines de Paris, 60, boulevard Saint-Michel, 75272 Paris Cedex 06, France
}

Received 14 May 2000; received in revised form 28 July 2000; accepted 20 September 2000

\begin{abstract}
We detail here the controller of the acid strength that we implemented in the Elf-Antar France refinery in Feyzin (France). The control technique used is new. It relies on the flatness property of the system to solve a constrained minimum time objective as successive linear-programming problems. This controller is in full service since January 1997. We detail the control technique, including the estimation and numerical problems and then give industrial results over 6 months. (C) 2001 Elsevier Science Ltd. All rights reserved.
\end{abstract}

Keywords: Alkylation unit; Process control; Optimization

\section{Introduction}

The alkylation of butenes is a common operation in oil refineries. It allows the synthesis of an interesting product, suitable to enter the composition of gasolines: the alkylate has a good octane number. Many kinds of units exist, but we concentrate here in the unit operated at the Elf Antar France's Feyzin refinery, which uses sulfuric acid as a catalyst.

The acid catalyst feeds two reactors in series. This feed is continuous. Partially destroyed during alkylation, the catalyst is withdrawn from the second reactor to feed a storage tank for off-site regeneration. A minimum amount of catalyst must be provided for the reactors to operate correctly. Providing more catalyst than the required minimum decreases the risks. But this implies expensive over-consumptions. The operator then tries to stabilize the unit just above the minimum. But the deterioration of the catalyst is very slow, and this makes such a manual driving difficult.

In 1996, the refinery decided to install a controller in order to limit acid consumption. The unit being very slow, we have decided to implement a minimum time control algorithm, applying results of a current

\footnotetext{
* Corresponding author
}

collaboration between Elf and the "Centre Automatique et Systèmes" of the École des Mines de Paris. This controller is being used since January 1997, with a service factor higher than $98 \%$. Under similar unit environments, it brings about $5 \%$ savings, which corresponds to a return time on investment of approximately 6 months.

\section{Process description}

The alkylation is made of two principal flow paths displayed in Fig. 1: a flow path for hydrocarbons and another for acid. The unit organizes the reaction of butenes and iso-butanes to form iso-octanes. Flows, either mainly containing butenes (olefins) or isobutane (recycle), are mixed before feeding two reactors in parallel, where the reaction takes place, catalyzed by sulfuric acid. The product of the reaction is flashed. The gas phase is condensed to generate a cold recycle. Mixed with the olefins and recycle, it helps to compensate for the exothermicity of the reaction. The liquid phase is washed before feeding a deisobutanizer. Propane is inert and accumulates in the unit. It is withdrawn after the flash. At its top, the deisobutanizer concentrates the isobutane, either coming from the so-called saturated feed or remaining in the liquid phase of the flash. The bottom 


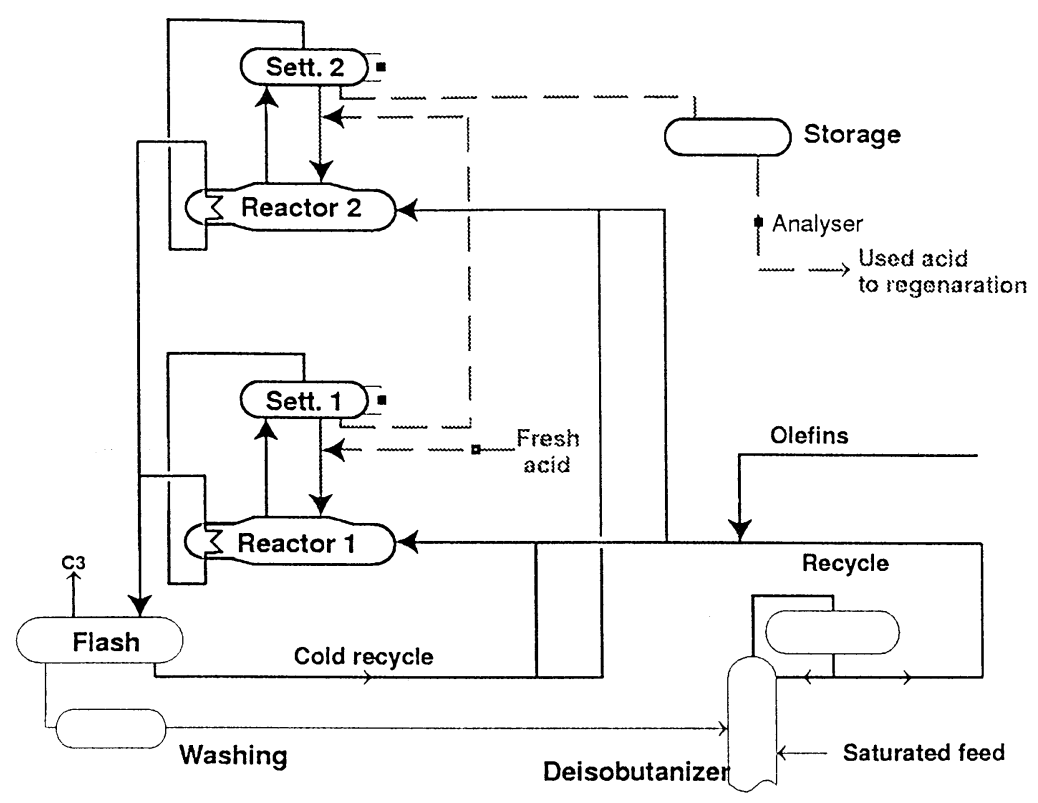

Fig. 1. Alkylation process.

product of this column essentially contains the alkylate and normal-butane (another inert), separated downstream.

The acid path flow is organized in series for the two reactors. A fresh acid flow (that is to say with a large acid concentration) feeds the first reactor and circulates between it and an associated settler. Secondary effects as reactions with impurities coming in small amounts with a flow of hydrocarbons induce a deconcentration of the acid (referred to as acid consumption). A flow, at a rate equivalent to the fresh acid flowrate, is withdrawn from the first settler and feeds the second reactor where a similar circulation is implemented with an associated settler. The second reactor also induces acid consumption. The used acid is withdrawn from the second settler and enters full storage tank. The acid from this tank is regenerated off-site. The concentration is analyzed at its output: this provides information about the nature of the forthcoming regeneration.

The fresh acid flowrate must be tuned to compensate for the variations of the acid consumption. Under a minimum concentration threshold, undesired reactions become important and induce serious malfunctions that must be avoided. Due to the way the unit is built, the concentration is the lowest at the output of the second settler. If its value is kept correct, good operating conditions are guaranteed for the two reactors. But allowing large security margins implies a large fresh acid flowrate that increases operating costs. It is better to work near the required minimum.

The slow variations of the acid concentration characterize this unit. A modification of the fresh acid flowrate is fully transmitted after about 1 week. Such a modification furthermore implies different residence times in the storage tank: they roughly vary from 8 to $24 \mathrm{~h}$.

\section{The control problem}

The acid flow path is first modeled. A linear model sufficient for control purposes is derived from this physical model. It is better to control the output concentration of the second settler rather than the measured output concentration of the storage tank. But this implies the construction of an estimator, because of the location of the analyzer. We also use the physical model for this purpose.

\subsection{Modeling}

The acid flow path is viewed as two blocks in series followed by a full storage tank considered as an ideal plug flow reactor. Each block consists in a reactor and a settler. It is considered as a perfectly mixed reactor, which is a sounded assumption. The acid flow path is then made of the concatenation of the two block models and the model for the storage tank.

For each block, we consider the partial mass variation of sulfuric acid:

$\rho V \frac{\mathrm{d} x}{\mathrm{~d} t}=-(u+A(p)) x+u x_{i n}$ 
where $\rho$ is the acid density (mass/volume), assumed constant, $V$ is the volume of acid phase in the block, assumed constant, $x$ is the acid mass fraction in the block. As the block is perfectly mixed, it is the output concentration, $x_{i n}$ is the acid mass fraction of the input flow, $u$ is the input and output flowrate, and $A$ is a consumption term. It depends on a set of 12 disturbances $p$ and takes into account all the effects implying a measurable acid deconcentration.

The storage tank is considered as a plug flow reactor. Dynamically, this tank introduces in the system a delay that is equal to the ratio between the mass of the acid contained in the tank and the flowrate. Finally, the model is

$$
\begin{aligned}
& \rho V_{1} \frac{\mathrm{d} x_{1}}{\mathrm{~d} t}=-\left(u+A_{1}(p)\right) x_{1}+u x_{f}, \\
& \rho V_{2} \frac{\mathrm{d} x_{2}}{\mathrm{~d} t}=-\left(u+A_{2}(p)\right) x_{2}+u x_{1}, \\
& y=x_{2}\left(t-\frac{K}{u}\right)
\end{aligned}
$$

where, $x_{1}$ and $x_{2}$, respectively, denote the sulfuric acid mass fractions at the output of the first and second blocks, $u$ is the control, that is to say the acid flowrate feeding the first reactor, $x_{f}$ is the sulfuric acid mass fraction of the fresh acid flow, $A_{1}$ and $A_{2}$, respectively, represent the acid consumptions in the first and second blocks, $\rho$ is the acid density, $V_{1}$ and $V_{2}$, respectively, represent the acid phase volumes in the first and second blocks, $y$ is the measured output. The dependency of delay on the control is denoted by $K / u$.

\subsection{Control design}

\subsubsection{Control model}

To act as efficiently as possible, we control an estimation of $x_{2}$. As $y$ corresponds to the delayed value of $x_{2}$, if $x_{2}$ is correctly controlled, so is $y$. We shall see in the sequel how the physical model is used to build an estimation of $x_{2}$. Because of the very slow dynamics, when the situation is analyzed on a time range of a few hours, it is possible to ignore the drift of the system and summarize information for the control in the linear approximation

$\frac{\mathrm{d}}{\mathrm{d} t}\left(x-x^{\text {mean }}\right)=a\left(u-u^{\text {mean }}\right)+\pi-\pi^{\text {mean }}$,

we use as a model and where

- $x$ is the acid mass fraction at the output of the second block and $x^{\text {mean }}$ its average value (we simplify notations: $x$ corresponds to $x_{2}$ in the previous sections),

- $u$ is the control and $u^{\text {mean }}$ its average value,
- $\pi$ denotes the contribution of measured disturbances and $\pi^{\text {mean }}$ its average value,

- $a$ denotes the gain by time unit.

Or, denoting, $P=-a u^{\text {mean }}+\pi-\pi^{\text {mean }}$ and considering that $x^{\text {mean }}$ is constant,

$\frac{\mathrm{d} x}{\mathrm{~d} t}=a u+P$.

The gain $a$ and the value of $P$ are computed from a tangent approximation of the physical model (initial slope of a step response).

\subsubsection{Minimum time constrained control}

3.2.2.1. Flatness property and control algorithm. The idea relies upon the possibility to explicitly parameterize via $x$ all the trajectories of the system. According to Fliess, Levine, Martin, and Rouchon (1995) and Martin, Murray, and Rouchon (1997), the system is flat and $x$ is its flat output. Assuming $x$ is known, $u$ is derived immediately. Constraints on $x$, on the control and its variations are all linearly expressed with respect to $x$. Discretizing the model, we are led to the question of existence of solutions for a linear-programming problem. In case of multiple solutions, we choose the one allowing $x$ to reach its setpoint in a minimum time.

Let us denote by $x^{i}$ the values of $x$ at the $n-1$ future sampling times and express the constraints that must be fulfilled over this horizon, exponent 1 denoting the current value. The $n$ constraints on $x$ are $x^{\min } \leqslant x^{i} \leqslant x^{\max }$. The $n-1$ constraints on $u$ are $a \Delta u^{\mathrm{min}}+\Delta P^{i} \leqslant$ $x^{i+1}-x^{i} \leqslant a \Delta u^{\max }+\Delta P^{i}$, where $\Delta$ is the sampling period and $P^{i}$ the contribution of disturbances at time $i$ (a constant equal to $P^{1}$ if no information is available about future disturbances). The sign of $a$ impacts these inequalities. Here it is strictly positive. Constraints on the variations of $u$ lead to similar expressions. Reaching the setpoint as an equilibrium point is achieved owing to the constraints $x^{n}=x^{n-1}=x^{\text {setpoint }}$. Finally, the current value $x^{\text {mes }}$ of $x$ (or its estimation in our case) is taken into account by $x^{1}=x^{\mathrm{mes}}$. All the constraints are summarized by $A X \leqslant B$, where $X$ is the vector of the $x^{i}$. Every $X$ obeying this inequality allows the construction of an admissible control profile:

$u^{i}=\frac{x^{i+1}-x^{i}-\Delta P^{i}}{a \Delta} \quad \forall i=1, n-1$.

As many solutions might exist for $X$, we must find a way to get a unique solution. We use a dichotomy on $n$ to find the vector $X$ with the lowest dimension that satisfies all the constraints. This is a minimum time control. Other approaches are possible. Only $u^{1}$ is applied and all the operations are computed at each sampling times, to partially compensate for nonmeasurable disturbances and 
modeling errors. At each sampling time, we also compute a prediction for the next time:

$x^{\text {pred }}=x^{\text {mes }}+\Delta a\left(u-u^{\text {mean }}\right)+\Delta\left(\pi-\pi^{\text {mean }}\right)$.

The filtered difference between the prediction and the "measure" is added to $P$. This is a standard compensation method.

3.2.2.2. Generalization. The idea of this method was originally described in Petit (1996). Note that it might be extended to (controllable) multivariable linear systems. This brings an alternative formulation of the classical linear predictive control algorithms (Richalet (1993)). This method lies in a natural framework for efficient nonlinear extension of these algorithms, namely the flatness framework (Fliess et al., 1995; Martin et al., 1997). When a system is flat, it is possible to directly work on its parameterized trajectories and doing so to avoid solving ordinary differential equations, that penalizes nonlinear predictive control and other approaches in dynamic optimization.

3.2.2.3. Numerical solving. To solve the successive linear programming problems we used a standard commercial simplex-based algorithm. Though these algorithm are known to have a nonpolynomial complexity (Klee \& Minty, 1972), it has been noted by many authors and specialists that in practice they behave very well and are very robust numerically speaking (Nering \& Tucker, 1993). This robustness combined to the breadth of the commercial packages and the relative low dimension of the problem led us not to consider interior-point algorithms.

\subsection{Estimation}

The control law described above assumes that $x_{2}$ is known. But only $y$ is measured: we have to construct an estimation of $x_{2}$ based on delayed measurements, furthermore with variable delays. We have tested many approaches before finding the following satisfactory answer:

$\rho V_{1} \frac{\mathrm{d} z_{1}}{\mathrm{~d} t}=-\left(u+A_{1}(p)\right) z_{1}+u x_{f}$

$\rho V_{2} \frac{\mathrm{d} z_{2}}{\mathrm{~d} t}=-\left(u+A_{2}(p)\right) z_{2}+u z_{1}$,

$y^{\prime}=z_{2}+\varphi$,

$\dot{\varphi}=\operatorname{sat}\left(z_{2}\left(t-\frac{K}{u}\right), y\right)-\frac{\varphi}{\tau_{f}}$,

where the estimated state is $z, \varphi$ denotes a filter of the difference between the delayed observation and the measured value of $y$. The first two equations of this system are a copy of those of the original model. For confidential reasons it is not possible to describe the sat function which is roughly speaking a linear saturated function.
Strictly speaking, it is possible to prove that if the system is not perturbed the $z_{1}$ and $z_{2}$ converge to $x_{1}$ and $x_{2}$. Though the system is time-varying, its triangular form allows to prove that $z_{1}$ tends exponentially to $x_{1}$ since $\left(u+A_{1}(p)\right)$ is lower-bounded by a positive constant. Then one can prove that $z_{2}$ converges exponentially to $x_{2}$ since $\left(u+A_{2}(p)\right)$ is lower-bounded by a positive constant and $\left(z_{1}-x_{1}\right)$ is an exponentially decreasing function (see, for instance, Khalil, 1992).

It should have been possible to use the classical highgain observer approach (see, Gauthier, Hammouri, \& Othman, 1992). But here the perturbations prevented us from implementing it successfully.

\subsection{Robustness}

Strictly speaking, we cannot prove the overall mathematical robustness of our approach.

On the one hand, the above observer gives good results, despite the perturbations, and is robust. Practically, this method insures that $y^{\prime}$ converges to $x_{2}$.

On the other, the control algorithm we use is numerically robust.

To show the experimental robustness of our approach are shown in Fig. 2 industrial results over 6 months with our controller.

\subsection{Implementation}

Implementing this control law took about 6 months. It constitutes a fast transfer between academic work and application in industry. The Feyzin refinery and the Elf research center have first developed and validated the model (we thank MM. Dajczman -Feyzin- and Djenab -CRES- for their fruitful participation). Together, we have then adapted to this problem the first results of N. Petit's Ph.D. thesis, detailed in Petit (1993). Finding a good estimator has revealed more time-consuming, because of the need for robustness in face of inaccuracies on variable delays.

The algorithm runs on a HP1000 computer. Its execution period is $15 \mathrm{~min}$.

\section{Conclusions}

The controller was rapidly accepted by the operators. Since its implementation, it has been used almost fulltime (service factor higher than 98\%). The operator have first observed the way it was working with a setpoint above the final objective. They were convinced by its ability to safely react in order to stabilize the unit (modifying the fresh acid flowrate before variations on the measured concentration was surprising at the beginning). After a short period, they accepted to decrease the setpoint, then decreasing the required fresh acid flow 


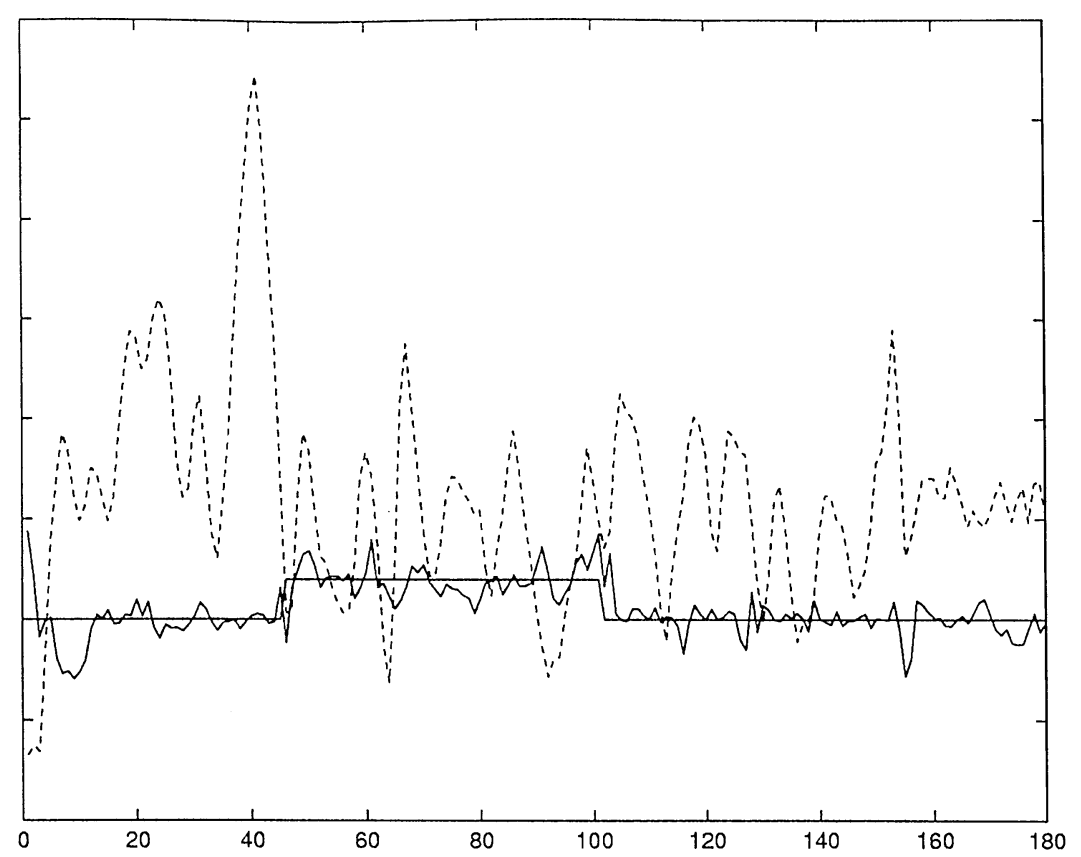

Fig. 2. Daily averages over 6 months: setpoint, result with our controller, and without it in dashed line (from history).

rate. Results of our controller over a period of 6 months are shown in Fig. 2. One can compare these results with results over a similar period of 6 months without our controller.

The benefits of this implementation are as follows. Stabilizing the unit allows the operators to concentrate on more difficult tasks. Furthermore, limiting acid consumption brings about $5 \%$ savings on the costs associated with the use of sulfuric acid.

\section{Appendix}

In the following, we show how to turn a solution to the discrete time optimization problem into a solution to a continuous time problem. This regularization is achieved owing to a convolution with a $C^{\infty}$ kernel and a time scaling (details about the classical technique of regularization can be found in Schwartz (1973, pp. 165-167)). This demonstrates Proposition 1.

Next, we show (Propositions 4 and 5) that both continuous and discrete time problem have a unique minimum time solution.

In the end, we conclude (Theorem A.1) that when the time step decreases to zero, the solution of the discrete time problem tends towards the solution of the continuous time problem.

Notation A.1. Given a set of real numbers $Y_{\min }^{(0)}, Y_{\max }^{(0)}$, $Y_{\max }^{(1)}, Y_{\max }^{(2)}$, where we assume $Y_{\max }^{(1)} \geqslant 0, Y_{\max }^{(2)} \geqslant 0$, let $C(T)$ be the subset of functions $Y \in C^{2}([O, T])$ satisfying the following conditions:

$C(T):\left\{\begin{array}{l}\forall t \in] 0, T[:, \\ Y_{\min }^{(0)} \leqslant Y(t) \leqslant Y_{\max }^{(0)}, \\ |\dot{Y}(t)| \leqslant Y_{\max }^{(1)}, \\ |\ddot{Y}(t)| \leqslant Y_{\max }^{(2)}, \\ Y(0)=0, \quad \dot{Y}(0)=0, \quad \ddot{Y}(0)=0, \\ Y(T)=1, \quad \dot{Y}(T)=0, \quad \ddot{Y}(T)=0 .\end{array}\right.$

Besides, let $D(N, \delta t)$ be the set of samples $Y_{N}=\left[Y_{N}(0), Y_{N}(1), \ldots, Y_{N}(N)\right]$ satisfying the following conditions:

$D(N, \delta t):\left\{\begin{array}{l}\forall i \in \mathbb{N}\left(\text { where if necessary } Y_{N}(i<0)=Y_{N}(0)\right), \\ Y_{N}(i>N)=Y_{N}(N): \\ Y_{\min }^{(0)} \leqslant Y_{N}(i) \leqslant Y_{\max }^{(0)}, \\ \left|\frac{Y_{N}(i+1)-Y_{N}(i)}{\delta t}\right| \leqslant Y_{\max }^{(1)}, \\ \left|\frac{Y_{N}(i+2)-2 Y_{N}(i+1)+Y_{N}(i)}{(\delta t)^{2}}\right| \leqslant Y_{\max }^{(2)}, \\ Y_{N}(0)=0, Y_{N}(N)=1 .\end{array}\right.$

In the following, $[x]$ denotes the largest integer less or equal to $x$.

Proposition A.1. $\forall(N, \delta t), D(N, \delta t) \neq \emptyset \quad$ implies $C((N+3 / 2) \delta t(1+\delta t)) \neq \emptyset$. 
Proof. Let $Y^{\text {aff }}$ be the function continuing the affine interpolation of $Y_{N}$ to the left and to the right of $[\delta t,(N+1) \delta t]$ :
$\left|\dot{Y}^{r}\right| \leqslant Y_{\max }^{(1)}$. The last formula directly implies that $\dot{Y}^{r}(0)=0$ and $\dot{Y}^{r}((N+1) \delta t+\varepsilon / 2)=0$.

$$
Y^{\mathrm{aff}}= \begin{cases}Y_{N}(0) & \text { if } t<\delta t, \\ Y_{N}(i-1)+\frac{Y_{N}(i)-Y_{N}(i-1)}{\delta t}(t-i \delta t) & \text { with } i=[t / \delta t] \text { if } \delta t \leqslant t<(N+1) \delta t \\ Y_{N}(N) & \text { if } t \geqslant(N+1) \delta t .\end{cases}
$$

Let $\chi_{\varepsilon}$ be an approximation to the unit, i.e., a positive function, the support of which is $[-\varepsilon / 2, \varepsilon / 2]$ and such that $\int_{-\varepsilon / 2}^{\varepsilon / 2} \chi_{\varepsilon}(s) \mathrm{d} s=1$.

We regularize $Y^{\text {aff }}$ into $Y^{r}$ by the following convolution:

$$
Y^{r}=Y^{\mathrm{aff}} * \chi_{\varepsilon} .
$$

The support of $Y^{r}$ is $[\delta t-\varepsilon / 2,(N+1) \delta t+\varepsilon / 2]$.

Let us assume that $0<\varepsilon / 2 \leqslant \delta$.

\section{Lemma A.1.}

$$
\begin{aligned}
& Y^{r}(0)=0, \quad Y^{r}\left((N+1) \delta t+\frac{\varepsilon}{2}\right)=1, \quad \text { and } \\
& \forall t,\left|Y^{r}(t)\right| \leqslant Y_{\max }^{(0)} .
\end{aligned}
$$

Proof. $\left|Y_{\text {aff }}\right| \leqslant Y^{\max }$ and $Y^{r}=Y^{\text {aff }} * \chi_{\varepsilon}$, then $\left|Y^{r}\right| \leqslant Y_{\max }^{(0)}$. At last, $\quad Y^{r}(0)=0$ since $\quad Y^{\text {aff }}(t<\delta t)=0$. Likely, $Y^{r}((N+1) \delta t+\varepsilon / 2)=1$ since $Y^{\text {aff }}(t>(N+1) \delta t)=1$.

\section{Lemma A.2.}

$\dot{Y}^{r}(0)=0, \quad \dot{Y}^{r}\left((N+1) \delta t+\frac{\varepsilon}{2}\right)=0, \quad$ and $\forall t,\left|\dot{Y}^{r}(t)\right| \leqslant Y_{\max }^{(1)}$.

\section{Proof.}

$$
\begin{aligned}
\dot{Y}^{r}(t)= & \dot{Y}^{\mathrm{aff}} * \chi_{\varepsilon}(t) \\
= & \frac{Y_{N}(i-1)-Y_{N}(i-2)}{\delta t} \int_{-\varepsilon / 2}^{\eta} \chi_{\varepsilon}(s) \mathrm{d} s \\
& +\frac{Y_{N}(i)-Y_{N}(i-1)}{\delta t} \int_{\eta}^{\varepsilon / 2} \chi_{\varepsilon}(s) \mathrm{d} s,
\end{aligned}
$$

where $\eta \in[-\varepsilon / 2, \varepsilon / 2], i=[t / \delta t]$, and $Y_{N}(i<0)=Y_{N}(0)$ $Y_{N}(i>N)=Y_{N}(N)$ if necessary.

$\dot{Y}^{r}(t)$ can be seen as the barycentre of $\left(Y_{N}(i-1)-\right.$ $\left.Y_{N}(i-2)\right) / \delta t \quad$ and $\quad\left(Y_{N}(i)-Y_{N}(i-1)\right) / \delta t$. Thus,
Lemma A.3. One may choose $\chi_{\varepsilon}$ such as

$$
\begin{gathered}
\ddot{Y}^{r}(0)=0, \quad \ddot{Y}^{r}\left((N+1) \delta t+\frac{\varepsilon}{2}\right)=0, \\
\text { and } \quad \forall t\left|\ddot{Y}^{r}\right| \leqslant(1+\delta t) Y_{\max }^{(2)} .
\end{gathered}
$$

\section{Proof.}

$$
\begin{aligned}
\dot{Y}^{r}(t)= & \dot{Y}^{\mathrm{aff}} * \dot{\chi}_{\varepsilon}(t) \\
= & \frac{Y_{N}(i-1)-Y_{N}(i-2)}{\delta t} \int_{-\varepsilon / 2}^{\eta} \dot{\chi}_{\varepsilon}(s) \mathrm{d} s \\
& +\frac{Y_{N}(i)-Y_{N}(i-1)}{\delta t} \int_{\eta}^{\varepsilon / 2} \dot{\chi}_{\varepsilon}(s) \mathrm{d} s \\
= & -\chi_{\varepsilon}(\eta) \frac{Y_{N}(i)-2 Y_{N}(i-1)+Y_{N}(i-2)}{\delta t},
\end{aligned}
$$

where $\eta \in[-\varepsilon / 2, \varepsilon / 2], i=[t / \delta t], Y_{N}(i<0)=Y_{N}(0)$ and $Y_{N}(i>N)=Y_{N}(N)$ if necessary. This yields

$\left|\ddot{Y}^{r}\right| \leqslant\left|\chi_{\varepsilon}(r)\right| Y_{\max }^{(2)} \delta t$

and

$\ddot{Y}^{r}(0)=0$,

$\ddot{Y}^{r}\left((N+1) \delta t+\frac{\varepsilon}{2}\right)=0$.

Let us choose $\chi_{\varepsilon}$ such as $\chi_{\varepsilon} \leqslant(1+\varepsilon) / \varepsilon$, which is compatible with $\int_{\varepsilon / 2}^{\varepsilon / 2} \chi_{\varepsilon}(s) \mathrm{d} s=1$. Then choose $\varepsilon=\delta t$. This gives

$\left|\ddot{Y}^{r}\right| \leqslant(1+\delta t) Y_{\max }^{(2)}$.

In the end, let us use a time scaling to define $Y^{d}: Y^{d}(t)=Y^{r}(t /(1+\delta t))$.

Lemma A.4. According to the previous notations, $Y^{d} \in C\left(\left(N+\frac{3}{2}\right) \delta t(1+\delta t)\right)$. 
Proof. As shown by Lemmas A.1-A.3:

$Y_{\min } \leqslant Y^{d} \leqslant Y_{\max }$

$\left|\dot{Y}^{d}\right| \leqslant Y_{\max }^{1}$,

$\left|\ddot{Y}^{d}\right| \leqslant Y_{\max }^{(2)}$

and

$Y^{d}(0)=0$,

$\dot{Y}^{d}(0)=0, \quad \ddot{Y}^{d}(0)=0$,

$Y^{d}((N+3 / 2) \delta t(1+\delta t))=1, \quad \dot{Y}^{d}((N+3 / 2) \delta t(1+\delta t))=$ 0 ,

$\ddot{Y}^{d}((N+3 / 2) \delta t(1+\delta t))=0$.

Finally, the support of $Y^{r}$ is $\left[\delta t / 2,\left(N+\frac{3}{2}\right) \delta t\right]$. This means that the support of $Y_{d}$ is included into $[0,(N+3 / 2) \delta t(1+\delta t)]$.

Lemma A.4 gives the conclusion of Proposition A.1.

Proposition A.2. $\forall T, C(T) \neq \emptyset \quad$ implies $\quad \forall \Delta T \leqslant 0$, $C(T+\Delta) \neq \emptyset$.

Proof. Assume $C(T) \neq \emptyset$, then there exists $Y \in C(T)$. For all $\Delta>0$, let us continue $Y$ into $\hat{Y}$ :

$\hat{Y}= \begin{cases}Y(t) & \text { if } t \leqslant T, \\ Y(T) & \text { if } T<t \leqslant T+\Delta .\end{cases}$

Obviously, $\hat{Y} \in C(T+\Delta)$ which is not empty.

Proposition A.3. $C(T) \neq \emptyset \Rightarrow \forall N \in \mathbb{N}^{*}, D(N, T / N) \neq \emptyset$.

Proof. Assume $C(T) \neq \emptyset$, then there exists $Y \in C(T)$. Let $\delta t=T / N$. Consider $Y_{N}=[Y(0), Y(\delta t), Y(2 \delta t), \ldots, Y(T)]$. In the following $Y_{N}(j)$ denotes the $(j+1)$ th coordinate of $Y_{N}$, the value of which is $Y(j \delta t)$.

(i) Obviously

$Y_{\min } \leqslant Y_{N}(j)=Y(j \delta t) \leqslant Y_{\max }$.

(ii) Let us consider the differences $Y_{N}(j+1)-$ $Y_{N}(j)=0+\delta t \dot{Y}\left(j \delta t+\theta_{j} \delta t\right)$ where $\left.\theta_{j} \in\right] 0,1[$ from MacLaurin's formula,

Yet

$\left|\dot{Y}\left(j \delta t+\theta_{j} \delta t\right)\right| \leqslant Y_{\max }^{(1)}$

so $\forall j, \quad\left|\frac{Y_{N}(j+1)-Y_{N}(j)}{\delta t}\right| \leqslant Y_{\max }^{(1)}$,

(iii) at last, let us consider the differences

$$
\begin{aligned}
& Y_{N}(j+2)-2 Y_{N}(j+1)+Y_{N}(j) \\
& \quad=Y((j+2) \delta t)-2 Y((j+1) \delta t)+Y(j \delta t)
\end{aligned}
$$

$$
\begin{aligned}
& =\delta t \dot{Y}((j+1) \delta t)+\frac{1}{2}(\delta t)^{2} \ddot{Y}\left((j+1) \delta t+\delta t \theta^{+}\right) \\
& \text {where } \left.\theta^{+} \in\right] 0,1[ \\
& -\delta t \dot{Y}((j+1) \delta t)+\frac{1}{2}(\delta t)^{2} \ddot{Y}\left((j+1) \delta t+\delta t \theta^{-}\right) \\
& \left.\quad \text { where } \theta^{-} \in\right]-1,0[ \\
& =\frac{1}{2}(\delta t)^{2}\left(\ddot{Y}\left((j+1) \delta t+\delta t \theta^{+}\right)+\ddot{Y}\left((j+1) \delta t+\delta t \theta^{-}\right)\right)
\end{aligned}
$$

which ends up in

$$
\begin{aligned}
& \frac{Y_{N}(j+2)-2 Y_{N}(j+1)+Y_{N}(j)}{(\delta t)^{2}} \\
& \quad=\frac{1}{2}\left(\ddot{Y}\left((j+1) \delta t+\delta t \theta^{+}+\ddot{Y}\left((j+1) \delta t+\delta t \theta^{-}\right)\right) .\right.
\end{aligned}
$$

Yet, as we already know

$\forall t, \quad|\ddot{Y}(t)| \leqslant Y_{\max }^{(2)}$

so

$\forall j, \quad\left|\frac{Y_{N}(j+2)-2 Y_{N}(j+1)+Y_{N}(j)}{(\delta t)^{2}}\right| \leqslant Y_{\max }^{(2)}$

In the end, Eqs. (A.1)-(A.3) ensure that $Y_{N} \in D(N, T / N)$ which is not empty.

Proposition A.4. There exists a unique minimum time, which we denote $T_{\operatorname{mini}}$, such as $C(T) \neq \emptyset$.

Proof. This is a direct conclusion from Proposition A.2.

Proposition A.5. For any given $\delta t$, there exists a unique minimum integer, which we denote $N_{\text {mini }}(\delta t)$ such as $D(N, \delta t) \neq \emptyset$.

Proof. The proof is similar to the one of Proposition A.2: let $Y \in D(N, \delta t)$, then it is clear that $[Y(0) Y(1) \ldots$ $Y(N) Y(N)]$ is an element of $D(N+1, \delta t)$.

Theorem A.1. The required time for the solution to the discrete time problem, $N_{\text {mini }}(\delta t) \delta t$, tends towards $T_{\operatorname{mini}}$ as $\delta$ tends towards zero. In other words, the discrete time problem tends to the continuous time problem as $\delta t$ tends towards zero.

Proof. From Proposition A.4, we know that there exists a unique minimum time $T_{\text {mini }}$ such that $C\left(T_{\text {mini }}\right) \neq \emptyset$. Given $\delta t$, one may write

$\left[\frac{T_{\operatorname{mini}}}{\delta t}\right] \delta t<T_{\operatorname{mini}} \leqslant\left(\left[\frac{T_{\operatorname{mini}}}{\delta t}\right]+1\right) \delta t$. 
This last equation means that

$C\left(\left[\frac{T_{\text {mini }}}{\delta t}\right] \delta t\right)=\emptyset$

$C\left(\left(\left[\frac{T_{\text {mini }}}{\delta t}\right]+1\right) \delta t\right) \neq \emptyset$.

Then, we deduce from Proposition A.3 that $D\left(\left[T_{\operatorname{mini}} / \delta t\right]+1, \delta t\right) \neq \emptyset$. Let $\delta t^{\prime}=(-1+\sqrt{1+4 \delta t}) / 2$, i.e., $\delta t^{\prime}\left(1+\delta t^{\prime}\right)=\delta t$. We must have $D\left(\left[T_{\operatorname{mini}} / \delta t\right]-\right.$ $\left.2, \delta t^{\prime}\right)=\emptyset$ otherwise, Proposition A.1 insures that $C\left(\left(\left[T_{\operatorname{mini}} / \delta t\right]-\frac{1}{2}\right) \delta t^{\prime}\left(1+\delta t^{\prime}\right)\right) \neq \emptyset$ which would mean that $C\left(\left[T_{\text {mini }} / \delta t\right] \delta t\right) \neq \emptyset$ which is not true as we know from Eq. (A.4). So

$$
\begin{aligned}
& D\left(\left[\frac{T_{\text {mini }}}{\delta t}\right]-2, \frac{-1+\sqrt{1+4 \delta t}}{2}\right)=\emptyset, \\
& D\left(\left[\frac{T_{\text {mini }}}{\delta t}\right]+1, \delta t\right) \neq \emptyset .
\end{aligned}
$$

Besides, as we know from Proposition A.5, for all $\delta t$ there exists a unique minimum integer $N_{\text {mini }}(\delta t)$ such that $D\left(N_{\text {mini }}(\delta t), \delta t\right) \neq \emptyset$.

The last two relations imply that:

$$
\begin{aligned}
& N_{\text {mini }}\left(\frac{-1+\sqrt{1+4 \delta t^{2}}}{2}\right) \leqslant\left[\frac{T_{\text {mini }}}{\delta t}\right]-2, \\
& N_{\text {mini }}(\delta t) \leqslant\left[\frac{T_{\text {mini }}}{\delta t}\right]+1
\end{aligned}
$$

We deduce that

$$
\begin{aligned}
& \lim _{\delta t \rightarrow 0} N_{\text {mini }}(\delta t) \delta t \leqslant T_{\text {mini }}, \\
& \lim _{\delta t \rightarrow 0} N_{\text {mini }}\left(\frac{-1+\sqrt{1+4 \delta t}}{2}\right) \delta t \leqslant T_{\text {mini }}
\end{aligned}
$$

which gives

$\lim _{\delta t \rightarrow 0} N_{\operatorname{mini}}(\delta t) \delta t=T_{\operatorname{mini}}$.

Solving the discrete time problem gives a solution to the continuous time problem owing to a regularization and a time scaling. The support of the obtained solution is $\left[0,\left(N_{\text {mini }}(\delta t)+\frac{3}{2} \delta t(1+\delta t)\right]\right.$ and it tends to $\left[0, T_{\text {mini }}\right]$.

\section{References}

Fliess, M., Lévine, J., Martin, Ph., \& Rouchon, P. (1995). Flatness and defect of nonlinear systems: introductory theory and examples. International Journal of Control, 61(6), 1327-1361.

Gauthier, J. -P., Hammouri, H., \& Othman, S. A. (1992). Simple observer for nonlinear systems - applications to bioreactors. IEEEtac, $37,875-880$.

Khalil, H. K. (1992). Nonlinear systems. New York: MacMillan.

Klee, V., \& Minty, G. J. (1972). How good is the simplex algorithm? In O. Shisha (Ed.), Inequalities, III (pp. 159-175), New York: Academic Press.

Martin, Ph., Murray, R. M., \& Rouchon, P. (1997). Flat systems. In Proceedings of the fourth European control conference (pp. 211-264). Plenary Lectures and Mini-courses.

Nering, E. D., \& Tucker, A. W. (1993). Linear programs and related problems. New York: Academic Press.

Petit, N. (1996). Systèmes $\delta$-libres sous contraintes. Rapport de stage de DEA, Université d'Orsay.

Richalet, J. (1993). Pratique de la commande prédictive. Hermès.

Schwartz, L. (1973). Théorie des distributions. Hermann. 